${ }^{3}$ Baker SP, O'Neill B, Haddon W, et al. The injury severity score: a method for describing patients with multiple injuries and evaluating emergency care. $\mathcal{F}$ Trauma 1974 ;14:187-96.

4 Bull JP. Measures of severity of injury. Injury 1978;9:184-7.

(Accepted 1 December 1983)

Accident and Emergency Department, University Hospital, Nottingham

GEOFFREY PYE, BM, MSC, senior house officer

ERIC A WATERS, BSC, FRCS, senior registrar

Correspondence to: Dr G Pye, senior house officer in general surgery, Derbyshire Royal Infirmary, Derby DE1 2QY.

\section{Quality control of home monitoring of blood glucose concentrations}

Home monitoring of blood glucose concentrations with reagent strips with or without a meter is widely used in the treatment of diabetics. The accuracy of the reagent strips and meters and the patients' ability to use them can be easily examined in the laboratory,
$(144 \mathrm{mg} / 100 \mathrm{ml})$. Doctors and specialist nurses were asked to rate their patients' expected performances on a scale of 0 to 10 ; the average score for each patient was then compared with their correlation coefficient (table) by Spearman's rank correlation.

A poor but significant relation existed between performance ratings and the rank correlation coefficients $(p<0.01)$. There were considerable differences between patients, however, and only two $(20 \%)$ patients using BM sticks gave satisfactory results compared with $11(78 \%)$ of those using Dextrostix and Glucochek (table). Close scrutiny of the individual results showed that poor readings occurred with both low and high glucose concentrations in patients using either monitoring method.

\section{Comment}

This small survey shows the need to review patients with unacceptably inaccurate readings and to check their equipment and method of estimating glucose concentrations to detect and eliminate the source of error. The visual acuity and colour sense of patients making visual readings should also be investigated. Errors may be caused by many things including unsatisfactory blood sampling, use of reagent strips after their expiry date, poorly calibrated meters, inappropriate handling of the strips, chemical contamination, and psychological factors.

To achieve better control of diabetes, therefore, we recommend the introduction of quality control for all patients who monitor their blood glucose concentrations at home.

We thank Richard Morris, department of community medicine, St Thomas's Hospital, for statistical advice.

Comparison of home and laboratory estimates of blood glucose concentrations in 24 patients

\begin{tabular}{|c|c|c|c|c|c|c|}
\hline \multirow{2}{*}{ Case No } & \multicolumn{3}{|c|}{ Compliance with filter paper technique } & \multirow{2}{*}{$\begin{array}{l}\text { Range of blood glucose } \\
\text { on filter paper } \\
(\mathrm{mmol} / \mathrm{l})\end{array}$} & \multirow{2}{*}{$\begin{array}{l}\text { Correlation } \\
\text { coefficient } \\
(\mathrm{r})\end{array}$} & \multirow{2}{*}{ Significance } \\
\hline & Unsatisfactory & Missed & Satisfactory & & & \\
\hline \multicolumn{7}{|c|}{$B M$ strips } \\
\hline $\begin{array}{r}1 \\
2 \\
3 \\
4 \\
5 \\
6 \\
7 \\
8 \\
9 \\
10\end{array}$ & $\begin{array}{r}0 \\
0 \\
0 \\
0 \\
3 \\
1 \\
1 \\
2 \\
0 \\
12\end{array}$ & $\begin{array}{l}2 \\
1 \\
0 \\
6 \\
0 \\
0 \\
0 \\
0 \\
0 \\
0\end{array}$ & $\begin{array}{r}12 \\
13 \\
14 \\
8 \\
11 \\
13 \\
13 \\
12 \\
14 \\
2\end{array}$ & $\begin{array}{l}2 \cdot 5-15 \cdot 0 \\
2 \cdot 6-14 \cdot 8 \\
6 \cdot 1-14 \cdot 4 \\
2 \cdot 1-18 \cdot 2 \\
3 \cdot 8-10 \cdot 5 \\
5 \cdot 5-18 \cdot 7 \\
3 \cdot 2-12 \cdot 0 \\
4 \cdot 7-13 \cdot 6 \\
1 \cdot 8-13 \cdot 8 \\
8 \cdot 8-19 \cdot 7\end{array}$ & $\begin{array}{l}0.89 \\
0.88 \\
0.94 \\
0.90 \\
0.87 \\
0.58 \\
0.97 \\
0.68 \\
0.69 \\
0.64\end{array}$ & $\begin{array}{l}\mathbf{p}<0.01 \\
\mathbf{p}<0.01 \\
\mathbf{p}<0.01 \\
\mathbf{p}<0.01 \\
\mathrm{p}<0.01 \\
\mathrm{p}<0.05 \\
\mathrm{p}<0.01 \\
\mathbf{p}<0.02 \\
\mathbf{p}<0.01 \\
\mathrm{p}<0.05\end{array}$ \\
\hline \multicolumn{7}{|c|}{ Dextrostix and Glucochek } \\
\hline $\begin{array}{l}11 \\
12 \\
13 \\
14 \\
15 \\
16 \\
17 \\
18 \\
19 \\
20 \\
21 \\
22 \\
23 \\
24\end{array}$ & $\begin{array}{l}0 \\
0 \\
2 \\
3 \\
1 \\
1 \\
6 \\
0 \\
0 \\
0 \\
0 \\
5 \\
8 \\
0\end{array}$ & $\begin{array}{l}0 \\
0 \\
0 \\
0 \\
0 \\
0 \\
2 \\
0 \\
0 \\
2 \\
0 \\
0 \\
0 \\
0\end{array}$ & $\begin{array}{r}14 \\
14 \\
12 \\
11 \\
13 \\
13 \\
6 \\
14 \\
14 \\
12 \\
14 \\
9 \\
6 \\
14\end{array}$ & $\begin{array}{l}2 \cdot 5-12 \cdot 1 \\
2 \cdot 4-19 \cdot 2 \\
4 \cdot 8-13 \cdot 8 \\
2 \cdot 7-18 \cdot 0 \\
2 \cdot 5-13 \cdot 4 \\
2 \cdot 3-15 \cdot 8 \\
2 \cdot 6-19 \cdot 9 \\
4 \cdot 4-18 \cdot 1 \\
2 \cdot 8-10 \cdot 8 \\
2 \cdot 7-15 \cdot 0 \\
2 \cdot 3-11 \cdot 2 \\
1 \cdot 9-15 \cdot 7 \\
3 \cdot 2-15 \cdot 0 \\
2 \cdot 8-12 \cdot 9\end{array}$ & $\begin{array}{l}0.94 \\
0.94 \\
0.87 \\
0.96 \\
0.96 \\
0.96 \\
0.96 \\
0.83 \\
0.96 \\
0.97 \\
0.92 \\
0.97 \\
0.91 \\
0.87\end{array}$ & 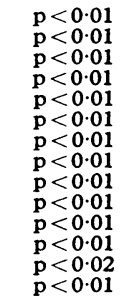 \\
\hline
\end{tabular}

Conversion: SI to traditional units-Blood glucose: $1 \mathrm{mmol} / 1 \approx 18 \mathrm{mg} / 100 \mathrm{ml}$.

but this gives no information about the quality of the patients' readings at home. We used blood samples on impregnated filter paper $^{12}$ to examine the reliability of patients' readings at home.

\section{Patients, methods, and results}

We asked 24 patients with more than one year's experience of home monitoring of blood glucose concentrations with either BM 20-800 strips (Boehringer Mannheim) or Dextrostix (Ames) in conjunction with the Glucochek reflectance meter (Medistron) to collect an additional blood sample on to filter paper (Whatman 31 ET CHR) each time they made their usual estimation of blood glucose concentration. They did this over two days obtaining a blood glucose concentration profile containing a total of 14 readings, and posted the filter paper strips with their readings to this hospital. Blood glucose concentrations in the filter paper spots were assayed enzymatically using an autoanalyser (Technicon), the within batch coefficient of variation of the method being $5.9 \% .^{2}$ The home readings of blood glucose concentrations were compared with the laboratory estimates using Spearman's rank correlation, and the correlation coefficient was calculated (table). We defined a satisfactory result as a set of six or more technically acceptable paired samples yielding a correlation coefficient greater than 0.9 over a range of blood glucose concentrations of $8 \mathrm{mmol} / \mathrm{l}$
1 Paisey RB, Bradshaw P, Hartog M, West P. Home monitoring of blood glucose using filter paper strips. Br Med $\mathcal{F} 1979$;ii:1509.

${ }^{2}$ Petranyi G. Home blood glucose sampling onto filter paper strips: a simple method for improving control of type-1 (insulin-dependent) diabetic patients. Diabetologia 1982;23:190-1.

(Accepted 1 December 1983)

Department of Medicine, St Thomas's Hospital Medical School, London SE1 7EH

G PETRANYI, MB, British Council research fellow

MARIA PETRANYI, MB, research assistant

I N SCOBIE, MB, MRCP, lecturer in medicine

P H SÖNKSEN, MD, FRCP, professor of endocrinology

Department of Clinical Chemistry, St Thomas's Hospital

R CRANE, FIMLs, senior chief medical laboratory scientific officer J ROBERTS, FIMLS, chief medical scientific officer

I S MENZIES, MB, FRCPATH, senior lecturer in chemical pathology

Correspondence to: Dr I N Scobie. 